\title{
WORK IN PROGRESS
}

"Work in progress" is a forum for students and university graduates who wish to present the results of their research.

Jacek Kos

University of Bialystok

\section{Anthropolinguistic Analysis of the Semantic Field of the Verb Cook}

\begin{abstract}
Anthropolinguistic analysis of the semantic field of the verb cook is an attempt at presenting the evolution of the domain of cooking in English as reflected in the vocabulary pertaining to cooking terms adopted in different historical epochs. The examination of the whole network of related cooking terms (i.e. the semantic field of to cook) proves that its development is inextricably linked with the cultural and social changes, new inventions, foreign influences and many other factors.
\end{abstract}

Keywords: anthropolinguistics, semantic field, to cook, semantic relations, evolution of mentality, analysis.

Semantic field is one of the most important notions in the domain of semantics. Crystal (1995: 157) defines semantic field as "a named area of meaning in which lexemes interrelate and define each other in specific ways", while Matthews (2003: 794) refers to it as "a distinct part of the lexicon defined by some general term or concept". It is an important object of study in anthropolinguistics. Anthropolinguistics is a relatively new science concerned with the development of human mentality and reasoning, as reflected in a language. It can be noticed that the way in which humans comprehended the reality varied in different periods in history. Three stages of the development of reasoning reflected in the language are usually distinguished: prescientific, proto-scientific and scientific. While the earliest stage was dominated by naïve type of thinking, the latest (i.e. scientific) period is based on the use of scientific theories and systems of concepts (Griniewicz in Griniewicz et al. 2004). The application of anthropolinguistic tools allows one to establish the changes in the development of English culture as illustrated by the evolution of the semantic field of to cook. 
As far as the English language is concerned, it is possible to distinguish three main periods of its development whose boundaries are a matter of convention. Each stage however, has its characteristic features. These periods are as follows:

- $\quad$ Old English (450 - 1150)

- Middle English (1150 - 1500)

- Modern English (1500 - present) (Baugh \& Cable 1991: 51-52).

More detailed divisions of the history of English into stages can also be found. It is common practice to divide Middle English period into Early Middle English (1100 - 1300) and Late Middle English (1300 - 1500) and Modern English into Early Modern English (1500 - 1800) and Late Modern English (1800 - present) (englishclub.com). Moreover, Late Modern English (1800 - present) is sometimes referred to as Present-Day English (Millward \& Hayes in Nordquist 2015). The present study will attempt to outline the evolution of the semantic field of the verb cook throughout these periods.

Using The Longman Synonym Dictionary (1992) and The Synonym Finder (1986) 21 lexemes within the field have been established: to bake, to roast, to broil, to charbroil, to grill, to barbecue, to sauté, to braise, to fricassee, to fry, to pan, to griddle, to toast, to boil, to simmer, to steam, to stew, to decoct, to brew, to poach, to blanch. The twenty-one lexemes together with the word to cook constitute one semantic field. The Oxford English Dictionary (1994) an online source Dictionary.com. provide some information regarding the stages in the development of the selected semantic field. The changes within the semantic field of to cook can be illustrated in the following way:

- Old English (450 - 1150)

to brew (893) (1)

to bake (1000) (1)

- Early Middle English (1100 - 1300)

to boil (1225) (1)

to fry (1290) (1)

to roast (1297) (1)

- $\quad$ Late Middle English (1300 - 1500)
to cook (1380) (1)
to broil (1386) (2)
to poach (1390) (1)
to blanch (1398) (2)
to stew (1420) (2)
to toast (1420) (2)
to griddle (1430) (1) 
- Early Modern English (1500 - 1800)

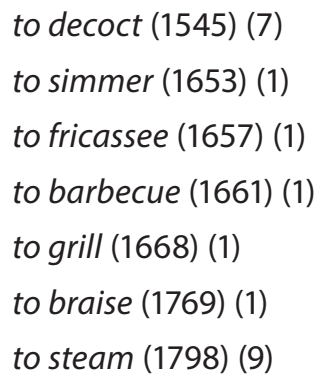

- $\quad$ Late Modern English (1800 - present)

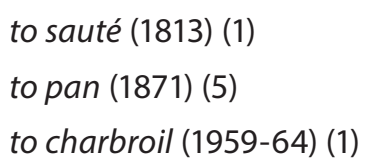

The dates presented in the list above indicate the year in which a particular lexeme was first attested with reference to food preparation. The number in the brackets denotes the number of meaning referring to cooking. Each of these lexemes appeared or adopted the meaning connected with cooking in different stages of the development of the English language.

What is noticeable in the analysis of the semantic field of to cook is the fact that in the case of certain verbs, e.g. to roast, their secondary meanings seem to precede their primary denotations. As far as the Old English period (450-1150) is concerned, it is clear that the domain of cooking was not particularly advanced as merely two verbs, i.e. to brew and to bake, referring to cooking techniques existed in English at that time. The verb to brew in the meaning 'to make (ale, beer and the like) by infusion, boiling and fermentation' (The Oxford English Dictionary 1994) was the one which initialized the semantic field of terms denoting cooking methods. Although, as it is implied by its meaning, the verb refers to preparing drinks, it can be included in the semantic field to cook as it involves processing items of food by heating them to make them ready for consumption.

The mentioned verb was the only one which referred to an action of preparing a drink. It might be expected that there should have existed a lexeme which indicated food preparation in a specific way. The verb to bake whose first usage was recorded in 1000 conveyed the following meaning: 'to cook by dry heat acting by conduction, and not by radiation, hence either in a closed place (oven, ashes, etc.), or on a heated surface, primarily used of preparing bread, then of potatoes, apples, the flesh of animals' (The Oxford English Dictionary 1994). Since the origins of the verb can be traced back to the Old High German form bachan, it may be assumed that the introduction of this lexeme is associated with Anglo-Saxon influence after their invasion on the British Isles in the 5th century AD.

Without doubt, the appearance of to brew and to bake must be regarded as a part of the initial stage of the domain of cooking and at the same time the semantic field to cook. The mentioned lexemes indicate rather different processes but both of them refer to preparing foodstuff by the use of 
heat. They might be qualified as co-hyponyms of another more general superordinate term which did not exist in the Old English period though.

The Middle English period (1150-1500), on the other hand, brought a significant broadening of the semantic field of the verbs denoting food preparation techniques by the use of heat. Thus, it is reasonable to expect numerous divisions in the field. The lexemes which already existed might have acquired new meanings. The overwhelming majority of cooking terms which appeared at that stage are of French origin. It can be treated as an outcome of the Norman conquest in 1066 which had an enormous impact on the British culture. As it turns out, the French influence is also visible in the domain of cooking.

In 1250, the verb to brew acquired another meaning which was 'to concoct, contrive, prepare, bring about, cause' (The Oxford English Dictionary 1994). As it can be inferred, the meaning was established on the basis of the resemblance to the primary meaning of the verb. In this way the lexeme became polysemous. Nevertheless, this change did not have any influence on the semantic field of cooking terms.

To fry is another verb which came into the lexical field under analysis in 1290. It conveyed the following meaning 'to cook food with fat in a shallow pan over the fire' (The Oxford English Dictionary 1994). This method of preparing meals without doubt differed significantly from the ones already known. The term indicating it might be perceived as a hyponym of a more general notion together with other already existing lexemes.

The verb to roast which appeared in the meaning 'to make (flesh or other food) ready for eating by prolonged exposure to heat at or before a fire' in 1297 (The Oxford English Dictionary 1994) contributed to the further division of the semantic field of cooking terms. Taking into consideration the definition of the mentioned lexeme, it might be pointed out that roasting was the first and most primitive method of preparing food as it did not involve any special skills or knowledge. Nevertheless, the lexeme did not appear as the first one but was preceded by to brew, to bake and to fry. Supposedly, there might have existed an earlier Old English counterpart of the verb to roast which was used even before the words to brew and to bake were coined. It might have been caused by the influence of French culture and language that the Old English lexeme has been ousted from the lexicon and replaced by the Norman equivalent to roast. It is worth mentioning that it existed some years earlier and its meaning was 'to torture by exposure to flame or heat' (1290). The new meaning must have developed on the basis of the similarity between the process of exposing food to fire and heating a person in a flame as a form of punishment. This association was possible due to the existence of cruel penal practices in the societies of medieval Europe.

The verb boil was first attested in 1325 and meant 'to cook solid articles by continued immersion in boiling liquid' (The Oxford English Dictionary 1994). It was the second lexeme after to brew which indicated cooking in water. It should be mentioned that the lexeme existed before in the following meanings 'to reach the boiling point, to turn from the liquid into the gaseous state' (1225) and 'to move with agitation like that of boiling water' (1300) (The Oxford English Dictionary 1994). Originally, the verb referred merely to water. Presumably, some similarity between a person mov- 
ing with agitation and the water undergoing the process of boiling based on their physical characteristics was noticed. Subsequently, with the development of reasoning, people figured out that it was possible to submerge food in the boiling water and make it ready for consumption in this way. Therefore, the process of boiling became more practical as it started to be utilized in preparation of many different items of food.

Not many years later, the verb to brew started to be used in the meaning 'to convert (barley, malt or other substance) into a fermented liquor' (1362) (The Oxford English Dictionary 1994). Clearly, the part of a larger process of brewing started to be referred by the same name as the whole process. The development of brewing industry might have been one of the reasons for such a transformation.

The verb to cook had not existed until the year 1380. It appeared then in the meaning 'to act as cook, to prepare food by the action of heat (for a household, etc.) (The Oxford English Dictionary 1994). Nevertheless, taking into consideration the literal meaning of the verb to cook, it can be inferred that the lexeme was not a general notion comprising all the existing lexemes denoting cooking techniques known at that time. The verb probably referred to preparing food as a professional activity performed by one person in a particular community. It might be assumed that there did not exist a cook as a job before the 14th century and food preparation was performed by any person.

The verb to fry used to mean 'to torture (a person) by fire; to burn or scorch' (The Oxford English Dictionary 1994) can be traced back to the year 1382. In this way the word became polysemous as the secondary meaning is related to the primary one. Nevertheless, the derived meaning refers to a different action not connected with food preparation. The appearance of the latter meaning did not have any impact on the semantic field to cook. There can be noticed a similarity between the verbs to roast and to fry whose secondary meanings are the same, i.e. 'to torture by exposure to flame or heat' and 'to torture (a person) by fire' respectively (The Oxford English Dictionary 1994). Comparing the primary definitions of the two mentioned verbs, it can be concluded that they refer to slightly different processes, i.e. frying involves cooking with extra fat while roasting does not. However, since they acquired identical metaphorical meaning, it might be assumed they were often used synonymously.

The $14^{\text {th }}$ century brought further broadening of the lexical field to cook. There appeared the verb to broil in the meaning 'to cook (meat) by placing it on the fire, or on a gridiron over it' (The Oxford English Dictionary 1994), first attested in 1386. Comparing the meanings of the two verbs, i.e. to roast and to broil, it can be concluded that most probably they were synonymous and used interchangeably in the Middle English period.

Roughly in the same period, the verb to bake acquired another meaning, i.e. 'to harden by heat' (1388) (The Oxford English Dictionary 1994). In this way the verb became polysemous. The word which was earlier used with reference to a cooking process acquired a related but rather different 
meaning. Later, in the Middle English period as well as in the following stage the verb became even more polysemous.

Another lexeme which appeared in the semantic field of to cook was the verb to poach. It was a completely new word which did not exist in English in any meaning earlier. As it has French roots in the form pochier and was without doubt incorporated into English as a result of Norman influences on British culture. Moreover, it referred to a very specific and sophisticated way of cooking. The meaning in which the verb appeared in 1390 was the following: 'to cook an egg by dropping it, without the shell, into boiling water simmering gently; to cook (fish, fruit, etc.) by simmering in water or another liquid' (The Oxford English Dictionary 1994). The lexeme with its meaning without doubt contributed to further divisions within the whole semantic field of to cook. It may have also limited the range of meanings of another already existing verb denoting cooking food in water, i.e. to boil.

The verb to blanch tracing its French roots in the form blanchir appeared in 1398. Its meaning which was 'to whiten almonds, or the like, by taking off the skin; to scald by a short rapid boil in order to remove the skin' (The Oxford English Dictionary 1994) was the first in which the word came into the lexicon. It became polysemous almost at the same time acquiring a more general but related meaning, i.e. 'to make white; whiten; to bleach' (1400) (The Oxford English Dictionary 1994). The mentioned case is a good illustration of a general tendency according to which more abstract notions gain their names from the domain of concrete terms evincing similar features.

To stew is another verb that broadened the semantic field to cook. Its meaning recorded in 1420 was the following: 'to boil slowly in a close vessel' (The Oxford English Dictionary 1994). It has its roots in the Old French form estui, which means 'case', 'sheath', etc. It already existed in the year 1400 in the meaning 'to bathe in a hot bath or a vapour bath' (The Oxford English Dictionary 1994).

The Middle English period is also the time when such verbs as to toast in its meaning to brown (bread, cheese, etc.) by exposure to the heat of a fire, etc.' (1420), to decoct in the meaning 'to prepare as food by the agency of fire' (1420) and to griddle in its meaning 'to cook on a griddle' (1430) entered the English lexicon (The Oxford English Dictionary 1994). It is difficult to explain the need for incorporating the verb to decoct in English, as there already existed lexemes referring to food preparation with the use of fire. It might have been treated as a hypernym of the terms such as to bake or to roast. The verb to griddle might have appeared as a result of the introduction of a new kitchen utensil, i.e. a griddle. Preparing food in it started to be referred to as griddling. The verb to bake, on the other hand, acquired two meanings, i.e. 'to prepare, make ready' (1460) and 'to form into a cake or mass' (1460) which are both obsolete now.

In the Modern English period (1500-1800), the verbs to brew, to bake, to poach, to blanch or to roast acquired new secondary meanings. The lexeme to bake started to refer to a more general process than preparing food. Its meaning recorded in 1527 was the following: 'to harden as frost does' (The Oxford English Dictionary 1994). The verb extended its polysemous character. Moreover, the word to blanch also acquired a new meaning related to its primary sense, i.e. 'to make pale with 
fear, cold, hunger, etc.' (1605) (The Oxford English Dictionary 1994). There is an evident visual similarity between making almonds white and a person becoming pale. It is clearly visible that the terms originating in the domain of cooking (e.g. to broil, to boil, to blanch) are frequently used in a metaphorical sense with reference to human emotions.

Although the term to decoct already existed in the Middle English, it appeared again in the subsequent stage in a more specialized meaning, i.e. 'to boil so as to extract the soluble parts or principles' (1545) (The Oxford English Dictionary 1994). It is difficult to judge whether its previous definition, i.e. 'to prepare food by the agency of fire' (1420) (The Oxford English Dictionary 1994) referred to a similar process or not due to its more general character.

The lexeme fricassee which appeared in the English lexicon in 1568 first operated as a noun denoting 'meat sliced and fried or stewed and served with sauce' (The Oxford English Dictionary 1994). The noun of an obvious French origin found its place in English as a result of an ongoing French influence on British culture. Later, it started to operate as a verb meaning 'to make a fricassee of; to dress as a fricassee' (1657) (The Oxford English Dictionary 1994). The process of conversion which is frequently encountered in the Modern English period is without doubt a very productive way of coining new words, as illustrated by the lexeme fricassee.

The appearance of the verb to cook (around 1611) meaning 'to prepare food; to make fit for eating by due application of heat' (The Oxford English Dictionary 1994) is an interesting fact. The term which is now regarded as the most general from the whole set of cooking terms did not initialize the mentioned semantic field. Presumably, due to the emergence of more and more cooking techniques together with the names referring to them, it was necessary to encompass them all under one notion.

The verb to grill which can be traced back to 1668 conveyed the meaning 'to broil on a gridiron or similar apparatus over or before a fire' (The Oxford English Dictionary 1994). Taking into consideration the verbs to broil, to roast and even to bake, it might be claimed that all of them could have been treated as synonyms at that time. They all refer to the process of exposing food to fire. Nevertheless, the definition of the verb indicates a specific device which is involved in the process of grilling. Presumably, with the introduction of more and more kitchen appliances and tools, the meaning of the verb to grill became specialized and the lexeme was no longer perceived as synonymous to other verbs indicating the process of heating food in a fire.

The acquisition of another meaning by the verb to stew seems to be an interesting phenomenon. The verb which previously indicated the process of cooking in a closed vessel appeared in the meaning 'to stay excessively long in bed' (1671) (The Oxford English Dictionary 1994). The change is likely to have been caused by the similarity between the duration of stewing as a cooking process and staying long in bed. This change in the meaning contributed to the polysemous character of the verb to stew. The lexeme turned out to be another term from the domain of cooking incorporated in a vocabulary segment representing another sphere of life.

The lexeme barbecue was another word which entered the semantic field to cook. In Present-Day English it operates both as a verb and a noun. Nevertheless, it entered the language as a verb in 
the meaning 'to broil or roast (an animal) whole' (1690) (The Oxford English Dictionary 1994) and then as a noun in the meaning 'a rude wooden framework used in America for supporting above a fire meat that is to be smoked or dried (1697) (The Oxford English Dictionary 1994). Taking into consideration the meaning of the verb, it might be assumed that before its emergence there already existed two words which referred to similar cooking processes, e.g. to broil or to roast. It is difficult to find a logical explanation for the emergence of another verb with a similar meaning. It might be partially explained by the fact that the word barbecue traces its roots in a Spanish form barbacoa. Presumably, it found its place in English thanks to the contacts between British and Spanish colonists in the North America. The verb to barbecue is more frequently encountered in American English which can be treated as a confirmation of the mentioned hypothesis. Moreover, the word appeared first as a verb and then as a noun which is quite unusual. Supposedly, as a loanword, it entered the English lexicon both as a verb and a noun since the first recorded usages (i.e. 1690, 1697) are not remote in time.

The Modern English period is characterized by the fact that many already existing words acquired new related meanings then. In the case of verbs, their new meanings usually referred to completely different processes. Nevertheless, there could be found certain physical similarities between the primary and secondary meanings. On the other hand, the verb to roast which is one of the lexemes which initialized the semantic field to cook acquired another meaning in 1724, i.e. 'to expose (coffee beans) to heat in order to prepare for grinding' (The Oxford English Dictionary 1994). The appearance of this new meaning had a direct influence on the semantic field to cook as the already existing term started to be used in reference to a new action. It might have been triggered by the popularization of coffee as a beverage among British people at that time.

Another term denoting a cooking method, i.e. to braise, found its place in the set of English vocabulary in the $18^{\text {th }}$ century (1797) with the meaning 'to stew in a tightly closed pan' (The Oxford English Dictionary 1994). The term referred to a complicated and sophisticated process which involved the meat being surrounded with slices of bacon, herbs, etc. The method and the term denoting it has been borrowed from French and therefore can be regarded as another indication of the cultural influence of France on the British Isles. Just like many other verbs indicating different ways of preparing food, the lexeme braise was known earlier as a noun. Its first usage was recorded in 1769 with the meaning 'braised meat' (The Oxford English Dictionary 1994).

Although the lexeme steam can be found in English as early as in the year 1000, it did not operate as a verb until the $18^{\text {th }}$ century (first recorded in 1798). It started to be used in the meaning 'to treat with steam for the purpose of cooking' (The Oxford English Dictionary 1994). As can be noticed, the previous meanings of the verb to steam (e.g. 'to be emitted or exhaled'), did not refer to any kind of cooking but rather to a physical process of emitting vapour or fume. Although steaming is quite an old way of preparing food, it may not have been referred to by this term at the earliest stages after its discovery. Similar to the lexemes griddle and grill, the verb to steam was first used as a noun and then started to operate as a verb too. Moreover, the year 1798, in which the verb to steam was first used in reference to food preparation, may have been an initial stage of 
healthy lifestyle involving popularization of cooking methods associated with retaining nutrients and departure from traditional cooking techniques based on fat and direct exposure to fire.

The modern English period is also characterized by further acquisition of new related meanings by the already existing verbs. The lexemes such as to bake, to toast and to roast which denoted cooking terms acquired the new meanings, i.e. 'to ripen with heat' (1697), 'to redden (by drinking)' (1701) and 'to criticize, to denounce' (1710) respectively (The Oxford English Dictionary 1994). However, the mentioned changes did not have a significant influence on the shape of the semantic field of to cook.

The appearance of new verbs denoting various cooking procedures which used to operate as nouns indicating certain kitchen utensils and appliances is one of the striking features of both the Modern and Present-Day English period. Food preparation with the use of griddles, pans and grills started to be referred as griddling, panning and grilling respectively.

The verb to simmer broadened the semantic field of to cook in 1823 with the meaning 'to keep in a heated condition just below boiling point' (The Oxford English Dictionary 1994). Obviously, the verb existed before but its meaning did not refer to the process of food preparation. Simmering was without doubt a cooking method unknown before and referred to preparation of new items of food such as different kinds of vegetables brought from the New World. The cooking technique which previously might have been encompassed by another term indicating cooking in water, i.e. to boil, started to be referred by a new separate term. The emergence of the verb to simmer divided the lexical field of to cook further and contributed to semantic shifts within it.

The verb to sauté appeared in English even earlier, i.e. in 1813. It refers to a specific way of cooking in which meat, vegetables, etc. are fried in a pan with a little butter over a high heat, while being tossed from time to time (The Oxford English Dictionary 1994). The lexeme is based on the French form sauter which means 'to jump'. Before the introduction of the verb into English, there already existed a verb with a very similar meaning, i.e. to fry. The verb to sauté might be perceived as partially synonymous to the verb to fry. On the other hand, it can also be claimed that frying is a more general term than sautéing and the relation existing between the two forms is one of hyponymy. Nevertheless, the introduction of the verb into the English lexicon broadened the semantic field of to cook and contributed to the emergence of the relations of hyponymy or synonymy while the terms indicating cooking in fat are concerned.

In Present-Day English, other lexemes previously denoting cooking terms acquired new meanings and therefore became polysemous. It might have been triggered by the evolution of human mentality or growing literacy in British society as the newly coined meanings of the already existing terms might seem to be poetic, e.g. to cook - 'to ruin, spoil, do for' (1851) or to grill - 'to subject to severe questioning' (1894) (The Oxford English Dictionary 1994). On the other hand, these verbs used in their secondary senses might belong to colloquial language which often incorporates words and phrases from the domains familiar for an average person (e.g. cooking).

The lexeme pan existed in English as early as in the 9th century and denoted 'a vessel of metal or earthenware for domestic uses' (897) (The Oxford English Dictionary 1994). Nevertheless, it is 
not possible to find the usage of the mentioned lexeme as a verb until the year 1871 in which it was first recorded in the meaning 'to cook or dress in a pan' (The Oxford English Dictionary 1994). Apparently, the word is far more frequently encountered as a noun than a verb. Nevertheless, the increasing popularity of metal kitchen utensils, e.g. pans, triggered the introduction of the verb to pan.

It can be concluded that the most polysemous verbs in the semantic field of to cook, i.e. to bake, to roast or to boil were the ones which were used the most frequently. Paying attention to this tendency may provide an answer to the question which cooking techniques were the most popular in different epochs. Even in the 20th century, the primary verbs in the mentioned lexical field, to bake and to cook, acquired new meanings, i.e. 'to be made uncomfortably hot (by the sun, a fire, etc.)' and 'to play music with excitement' respectively (The Oxford English Dictionary 1994).

Finally, the latest lexeme included in the semantic field of to cook is the verb to charbroil in the meaning 'to grill or broil over a charcoal fire' (Dictionary.com). It was coined in the years 195964 as a result of blending the two lexemes, i.e. charcoal and grill. Similar to panning, charbroiling must have been known earlier as a method of cooking although it was not referred to by the mentioned term then.

As already mentioned, the state of human mentality and reasoning in a certain period of time is reflected in the language (or vocabulary) used by the people at that time. As far as the domain of cooking is concerned, it is easy to notice that the ways of food preparation varied in different stages of human evolution. The development of the semantic field of to cook depended on many factors, such as the state of human reasoning, availability of particular items of food, cultural influences, changes in the lifestyle, etc. Taking into consideration the diachronic analysis conducted in the present thesis, it is possible to distinguish changes in the English mentality concerning the ways of cooking and define their possible reasons. It can be assumed that the advent of cooking, i.e. preparing food with the use of heat, is linked with the discovery of fire. Cooking should be without doubt regarded as one of the earliest human activities. Most anthropologists assume that fire was already controlled by the mankind 250,000 years ago (Wikipedia.org). On the other hand, the scientist Richard Wrangham supposes that the fire was known to humans as early as 1.8 million years ago and cooking began at nearly the same time (Wrangham 2009: 45). Due to the fact that archeological evidence is insufficient to establish the exact moment in which cooking started to be applied by the mankind, it is possible to search for clues in biological features of early humans (Wrangham 2009: 42). In the evolution of Homo erectus from habilines, it is possible to observe such transformations as the reduction in tooth and gut size, the increase in body size and a significant decline of climbing ability. They all may support the claim that cooking as a method of food preparation was already known to Homo erectus who appeared approximately 1.8 million years ago (Wrangham 2009: 46). Therefore, it seems legitimate to maintain that the emergence of cooking had an enormous impact on the further physical and mental development of human beings which facilitated the rise of modern civilization. 
Just like cooking prompted the progress of human mentality and reasoning, it should also be admitted that the emergent abilities of the mankind contributed to the introduction of more and more advanced cooking techniques. As it is assumed, roasting, inseparably combined with the use of fire, was the only method of preparing meals known to the earliest human beings. It turns out that steaming is also one of the earliest cooking methods as the Aurignacian people of southern France prepared their food in this way wrapping it in wet leaves. The introduction of pottery in the Neolithic period gave rise to the entirely new methods of preparing meals such as boiling, stewing or braising which diversified the domain of cooking. Additionally, the appearance of metal cookware gave an opportunity for a more frequent usage of cooking methods based on water. Social changes such as the development of settled communities, domestication of livestock and the beginning of cultivation of edible plants also contributed to the emergence of new cooking techniques (allthatcooking.com).

Taking into consideration the linguistic analysis of the semantic field of to cook, it is possible to conclude that it developed unevenly in different historical periods. As the data available in The Oxford English Dictionary (1994) suggest, the first lexeme which initialized the semantic field of cooking terms is the verb to brew. It appeared as early as the $9^{\text {th }}$ century AD which is the Old English period. It is unsurprising as brewing is one of the earliest cooking methods applied by people. It was already known to the inhabitants of ancient Egypt and Mesopotamia (Wikipedia. org). The method referred to by the term brewing was originally applied to making beer which suggests that beer was one of the first beverages consumed by ancient people. A small number of cooking terms existing in the Old English period, i.e. early Middle Ages, indicates that very few manners of preparing food were known to people living at that time. It might have stemmed from a rather low level of education of the majority of the population. Moreover, the early Middle Ages was a specific period in which spiritual values were propounded over material ones. In other words, there was no particular importance attached to the preparation of food, its taste or nutritious values.

Another lexeme referring to food preparation existing in the Old English period, i.e. to bake, has its roots in an Old High German form bachan. The time of the appearance of the form to bake in English is consistent with Germanic invasions on the British Isles which took place in the $5^{\text {th }}$ century. As the given evidence is concerned, Germanic (or Anglo-Saxon) culture did not contribute to the development of cooking habits applied by the population inhabiting the British Isles. It can be concluded that Anglo-Saxons were not particularly inventive in reference to cooking techniques.

The Middle English period, on the other hand, is characterized by the appearance of numerous verbs denoting different ways of preparing food with the use of heat. The introduction of many previously unknown cooking techniques might have been linked to the development of human reasoning or the emergence of new human needs concerning tastes of meals and nutritious values. However, the most probable reason for such a significant broadening of the semantic field of cooking terms in the Middle English period was the influence of another culture. After the Norman 
conquest in 1066, England was regarded mainly as a cultural colony of France. Such a stance is perfectly understandable if the influences in the fields of music, literature or architecture are taken into consideration (Morgan 1997: 107). It turns out that an overwhelming majority of cooking terms which were incorporated into English after the Norman conquest in 1066 such as to roast, to boil or to stew, which are of a French origin. It is also worth mentioning that a considerable number of terms denoting kinds of fish and meat, fruit, seasoning and condiments have French roots. However the impact of the invaders on the Anglo-Saxon culture might be perceived, it should be admitted that the Normans enriched the culture of the locals in the domain of cuisine in a significant way although all those new methods were adopted exclusively by the aristocracy who were willing to use the French language and cultivate Norman customs (Baugh \& Cable 1991: 171).

The Modern English period, which began approximately in 1500, can also be regarded as a phase of a dynamic development of the semantic field of cooking terms. A significant number of verbs denoting various cooking processes appeared in English at that time. Generally, the year 1500 is regarded as the beginning of the Renaissance. The most important inventions of this particular period in history such as the appearance of the printing press, the spread of popular education, new means of communication and the growth of social consciousness exerted an enormous influence on the evolution of English (Baugh \& Cable 1991: 199).

As far as the domain of cuisine is concerned, the introduction of new vegetables such as potato (1586), broccoli (before 1724) or tomato (1750s) after the discovery of the New World may have facilitated the rise of new methods of cooking so that these obscure items of food were fit for consumption (Wikipedia.org). The appearance of many new kitchen utensils may also have played a significant role in the evolution of cookery at that time. Although the metal cookware was present in earlier epochs, it was the $17^{\text {th }}$ century that brought popularization of brass, copper and iron cooking vessels. A typical Western kitchen of that time was equipped with skillets, baking pans, a kettle and several pots (Wikipedia.org). Moreover, due to the social changes in France in the 1790s, many chefs had to emigrate in order to find a job in various places in Europe. In this way, as the French are perceived as the first who created and wrote down the systems and rules for cooking, they instilled professional approach to cooking in various societies including the British (Clarke 2013). Thus, the verb to fricassee was created on the basis of a French noun fricassee denoting stewed meat served with sauce. Similarly, frying food quickly in hot fat started to be referred to as sautéing. The appearance of other cooking terms in English in the modern period can also be justified by social changes and discoveries in the fields of culture, science, etc.

In the case to steam, it is legitimate to assume that the fact of acquiring the meaning 'to treat with steam for the purpose of cooking' (The Oxford English Dictionary 1994) by it and the invention of the steam engine at nearly the same time were not coincidental. Presumably, it was observed that the produced steam can also be used in the process of cooking thanks to its heating properties. It might be useful to mention that steaming as a method of cooking can be regarded as one of the earliest (allthatcooking.com). Nevertheless, the process applied by prehistoric people 
involved exposing food wrapped in wet leaves to the steam from hot embers and thus it cannot be compared to the method of steaming employed in the present-day art of cooking.

The verb to brew appeared as early as the $9^{\text {th }}$ century and was used in reference to making beer. Nevertheless, in the Modern English period it acquired another meaning, i.e. 'to make by infusion, as tea' (1626) (The Oxford English Dictionary 1994). The period in which the mentioned verb started to be used with reference to the preparation of tea can be associated with the introduction of this beverage in England by a Portuguese woman Catherine of Braganza, wife of King Charles II. The tea did not gain a widespread popularity until the end of $18^{\text {th }}$ century though (Wikipedia.org).

Finally, to charbroil is the only lexeme included in the present analysis which came into existence in the meaning referring to food preparation in the Present-Day English period. It was coined as the result of blending two lexemes, i.e. charcoal and broil (Dictionary.com). Although the method of cooking does not seem to be one of the latest, it has been referred to by the mentioned term for only about fifty years so far. It might be explained by the general tendency of inventing new names for the already existing phenomena. Presumably, it is triggered by the rapid development of many scientific and cultural domains which results in the appearance of a significant number of new terms. The entities once referred to by more general notions acquire new more specific names.

To sum up, the evolution of human mentality and reasoning is reflected in particular stages of the development of a language. Taking into consideration the examples presented above, it should be concluded that cooking as one of the primary human activities has always been dependent on human mentality and reasoning. However, the advent of cooking facilitated physical and mental development of mankind and provided the foundations for the rise of modern civilization too. As the new methods of preparing food by the use of heat emerged, there were coined the lexemes denoting those cooking techniques. In this way, the semantic field 'to cook' had a chance to broaden its range and incorporate more and more terms. It would be reasonable to expect further development of the mentioned semantic field in the future as thanks to numerous technological improvements, there appear more and more kitchen utensils. Moreover, some verbs may disappear if certain cooking techniques come out of use due to various reasons. Nevertheless, the ongoing process of globalization will favour the introduction of different ingredients unknown in Anglo-American culture and thus the further broadening of the semantic field of to cook might be expected.

\section{References}

All that cooking. History of cooking.

http://allthatcooking.com/history-of-cooking/. Retrieved: 08.05.2015

Baugh, Albert C.; Cable, Thomas. 1991. A History of the English Language. London: Routledge.

Brewing - Wikipedia, the free encyclopedia

en.wikipedia.org/wiki/Brewing. Retrieved: 10.05.2015

British cuisine - Wikipedia, the free encyclopedia

en.wikipedia.org/wiki/British_cuisine. Retrieved: 08.05.2015 
Clarke, Lydia. 2013. Haute Cuisine: Why Is French the Lingua Franca of Cooking? http://www.acclaro.com/translation-resources/translation-localization-blog/haute-cuisine-355. Retrieved: 08.05.2015

Cooking - Wikipedia, the free encyclopedia en.wikipedia.org/wiki/Cooking. Retrieved: 10.05.2015

Crystal, David. 1995. The Cambridge Encyclopedia of the English Language. Cambridge: Cambridge University Press.

Dictionary.com. http://dictionary.reference.com/.

Grinev-Griniewicz, Siergiej, Particia Thomas and Sławomir Raube (eds). 2004. Language and Culture: establishing foundations for anthropological linguistics. Białystok: Wyższa Szkoła Finansów i Zarządzania.

Matthews, Peter H. 2003. The Concise Dictionary of Linguistics. Oxford: Oxford University Press.

Morgan, Kenneth O. (ed.). 1997. The Oxford Illustrated History of Britain. Oxford: Oxford University Press.

Nordquist, Richard. 2015. Present-Day English (PDE). http://grammar.about.com/od/pq/g/Presentday-English-pde.htm. Retrieved: 02.02.2015.

Rodale, Jerome I. 1986. The Synonym Finder. New York: Warner Books.

Simpson, John A. and Weiner, Edmund. 1994. The Oxford English Dictionary. Oxford: Clarendon Press.

Tea - Wikipedia, the free encyclopedia en.wikipedia.org/wiki/Tea. Retrieved: 08.05.2015

The Longman Synonym Dictionary. 1992. Urdang, Laurence (ed.). Harlow: Longman.

Wrangham, Richard. 2009. Catching Fire: How Cooking Made Us Human. New York: Basic Books. 